\title{
Caracterización mecánica de recubrimientos de aluminio por CVD-FBR sobre aceros inoxidables y resistencia a la oxidación en vapor de agua
}

\author{
Mechanical characterization by CVD-FBR aliminium coatings on \\ austenitic stainless steel and the steam oxidation resistance \\ Caracterização mecânica de recobrimentos de alumínio por CVD- \\ FBR sobre aços inoxidáveis e resistência à oxidação em vapor \\ de água
}

Diego Pérez-Muñoz

Fecha Aprobación: 15 de Agosto de 2015

José Luddey Marulanda-Arévalo** Juan Manuel Meza-Meza**

\section{Resumen}

Los recubrimientos de aluminio depositados sobre el acero inoxidable austenítico AISI 317 por Deposición Química de Vapor en Lecho Fluidizado (CVD-FBR) presentan a altas temperaturas una reducción de la velocidad de corrosión de más de 80 veces. Se realizó la caracterización mecánica de los recubrimientos por medio de microdureza, nanoindentación, para conocer cómo se vieron afectas las propiedades mecánicas (en especial la dureza y el módulo de Young) del recubrimiento y del sustrato luego de ser sometidos a la oxidación a alta temperatura. También se hicieron análisis por medio de Microscopia Electrónica de Barrido (MEB), para observar los cambios microestructurales, y de Microscopia de Fuerza Atómica (MFA), para observar cómo varía la topografía y el gradiente de rugosidad en función de la distancia recorrida por la punta del cantiléver durante los barridos.

Palabras clave: Deposición química de vapor, Recubrimientos de aluminio por CVD-FBR, Corrosión, Oxidación en vapor.

$\begin{array}{ll}* & \text { Universidad Tecnológica de Pereira (Pereira - Risaralda, Colombia). dperez@utp.edu.co. } \\ \text { ** } & \text { Ph.D. Universidad Tecnológica de Pereira (Pereira - Risaralda, Colombia). jlmarulanda@utp.edu.co. } \\ \text { *** } & \text { Ph.D. Universidad Nacional de Colombia (Medellín - Antioquia, Colombia).jmmezam@unal.edu.co. }\end{array}$ 


\begin{abstract}
The aluminum coatings deposited by Chemical Vapour Deposition Fluidisation (FBR-CVD) on the austenitic stainless steel AISI 317, show a more than 80 times corrosion rate reduction at high temperatures. The coatings mechanical characterization was conducted by means of microhardness, nano indentation, in order to konow how were affected the coating and the substrate's mechanical properties (particularly the hardness and the Young's modulus) after being subjected to oxidation at high temperature. Some analyses were also made by means of SEM, to observe the micro structural changes and to see how is the AFM topography and rugosity gradient variation, related to the distance traveled by the tip of the cantilever during the sweeps.
\end{abstract}

Keywords: Chemical vapour deposition, aluminum coatings by FBR-CVD, corrosion, steam oxidation, Hardness.

\title{
Resumo
}

Os recobrimentos de alumínio depositados sobre o aço inoxidável austenítico AISI 317 por Deposição Química de Vapor em Leito Fluidizado (CVD-FBR) apresentam a altas temperaturas uma redução da velocidade de corrosão de mais de 80 vezes. Realizou-se a caracterização mecânica dos recobrimentos por meio de microdureza, nanoindentação, para conhecer como foram afetadas as propriedades mecânicas (em especial a dureza e o módulo de Young) do recobrimento e do substrato após serem submetidos à oxidação a alta temperatura. Também foi feita a análise por meio de Microscopia Eletrônica de Varredura (MEB), para observar as mudanças microestruturais, e de Microscopia de Força Atômica (MFA), para observar como varia a topografia e o gradiente de rugosidade em função da distância recorrida pela ponta do cantiléver durante a varredura.

Palavras chave: Deposição química de vapor, Recobrimentos de alumínio por CVD-FBR, Corrosão, Oxidação em vapor. 


\section{INTRODUCCIÓN}

Los recubrimientos pueden ser el medio más rentable para proteger materiales contra el desgaste y la corrosión, además, pueden mejorar el desempeño mecánico del material. La confiabilidad y la eficiencia de las capas hacen necesario que estos recubrimientos sean bien seleccionados y correctamente aplicados para su buen funcionamiento. Estos hacen aumentar la vida útil de las componentes de las máquinas e instalaciones, lo que permitiría ahorrar gran parte del consumo mundial de materiales $[1,2]$. En el análisis para seleccionar el revestimiento y el proceso de aplicación se deben tener en cuenta factores como el costo y la eficiencia a mediano y largo plazo. En el mundo contemporáneo, en donde se busca mayor competitividad, todos los ojos se vuelven hacia dos factores de extrema relevancia: la alta productividad y el bajo impacto ambiental [3]. La ingeniería actual está en una situación de constante desafío, debido a la diversidad de opciones con relación al empleo de los materiales y a la competitividad industrial, lo que lleva a una compleja situación en la que se deben unir características de desempeño a factores de costo y proceso en la elección de los materiales más adecuados en una aplicación específica $[4,5]$.

Los recientes avances en las tecnologías de los recubrimientos permiten hoy depositar películas con propiedades que hace unas décadas eran inalcanzables. Técnicas como la Deposición Química de Vapor (CVD) y la Deposición Física de Vapor (PVD) son algunas de las que han estado a la vanguardia de estas novedades; estas permiten grandes resultados al diseñar y combinar excelentes sistemas capa/substrato $[5,6]$.

La Deposición Química de Vapor por Lecho Fluidizado (CVD-FBR) es una variante de la técnica de CVD que combina las ventajas de la activación térmica por calentamiento con el lecho fluidizado, aumentando la transferencia de masa y calor entre el gas, el lecho y las muestras inmersas dentro del reactor; esto permite tener mayor uniformidad en la temperatura y una muy buena mezcla de los gases reactivos con las partículas fluidizadas, lográndose un alto grado de reacción de todas las especies activadas en el lecho, ya que en la fluidización existe un excelente contacto entre las partículas sólidas y el medio de fluidización gaseoso [7-10]. Mediante la deposición por CVD-FBR se pueden recubrir piezas de grandes áreas, "in-situ”, lo que significa una ventaja desde el punto de vista económico.

Ahora bien, considerando un material en específico, con los recubrimientos de aluminio se puede aumentar la resistencia a la oxidación en vapor de agua de los aceros inoxidables austeníticos en más de 80 veces [5, $7,11]$, ya que generan una capa superficial protectora, compacta y adherente, de óxidos de aluminio que favorece la formación de fases ricas en cromo y níquel debajo del recubrimiento; además, el aluminio del recubrimiento se consume en dos formas: una parte se consume para formar la capa superficial de alúmina, y la otra parte difunde dentro del substrato durante la oxidación $[6,7]$. Siendo claras las ventajas de los recubrimientos de aluminio en contra de la corrosión a altas temperaturas, el siguiente paso es conocer qué cambios sufren el recubrimiento y el sustrato en cuanto a sus propiedades mecánicas; para ello se realiza la caracterización mecánica, buscando obtener la validez y confiabilidad suficiente de los recubrimientos de aluminio.

La caracterización mecánica de un material requiere, entre otros aspectos, el estudio de propiedades mecánicas, físicas, químicas y estructurales [12]. La relación entre la estructura del material y sus propiedades es un tema crítico para el funcionamiento deseado en una herramienta o dispositivo; es posible investigar esta relación a nivel de nanoescala, y de esta forma explorar las posibles conexiones entre la estructura y la funcionalidad de un material a escala nanométrica, centrándose en las propiedades mecánicas elásticas [13].

En particular para los materiales recubiertos que se estudiaron en este trabajo, los espesores de capa están del orden de unas pocas micras, por tal motivo se utilizaron dos de las técnicas más importantes para la caracterización mecánica: la microscopia de fuerza atómica (AFM, en inglés) y la nanoindentación. El microscopio de fuerza atómica es un instrumento capaz de detectar cambios en la superficie de un material en el orden de los nanómetros a través de la medición óptica del movimiento de un cantiléver sobre la superficie de una muestra. El cantiléver tiene una longitud de 200 micras y termina en una punta cónica, con sensibilidad entre $0.1 \mathrm{~nm}$ y $1 \mathrm{~nm}$. Esta técnica tiene resoluciones entre $0.1 \mathrm{~nm}$ y $1 \mathrm{~nm}$, y permite explorar una amplia gama de fenómenos 
físicos y químicos. Uno de los modos de trabajo de esta técnica es el denominado "Contrast Phase", que es capaz de proveer información topográfica, microestructural e, indirectamente, de composición química; la técnica se basa en hacer que la punta oscile e intermitentemente entre en contacto con la superficie por investigar. Se registran las señales de oscilación y se detectan las diferencias de amplitud, que dependen de las propiedades del material, en particular de la composición, reología y, además, de la topografía. Existen varias revisiones sobre esta técnica que pueden ser consultadas [14-16].

Por otra parte, la nanoindentación consiste en aplicar una fuerza mediante un indentador que penetra la superficie por investigar; es una prueba donde la escala de deformación está en el orden de los nanómetros $\left(10^{-9} \mathrm{~m}\right)$, por lo que se considera un ensayo de tipo no destructivo. A partir de los datos de carga y desplazamiento se pueden inferir la dureza del material, además de otras propiedades como el módulo elástico y la resistencia a la fractura [17-19]. Esta técnica es especialmente interesante para el estudio de materiales multifásicos y materiales recubiertos, ya que permite determinar la variación en las propiedades en volúmenes o áreas muy pequeñas, razón por la que será utilizada en este trabajo.

\section{Procedimiento experimental}

Se usaron probetas del acero inoxidable austenítico AISI 317, las cuales fueron maquinadas $(20 \mathrm{~mm}$ x $6 \mathrm{~mm}$ x $2 \mathrm{~mm}$ ) y lijadas desde papel esmeril N. ${ }^{\circ}$ 100 hasta N. ${ }^{\circ} 600$, para luego limpiarlas en un baño de acetona por ultrasonido durante 10 minutos. Los recubrimientos de aluminio fueron depositados usando el proceso de Deposición Química en Fase Vapor mediante Lecho Fluidizado (CVD-FBR) a presión atmosférica, y fueron analizados por microscopia electrónica de barrido (SEM) en un microscopio electrónico de barrido JEOL Mod. JM-6400. Dicho microscopio tiene acoplado un sistema de análisis de espectroscopía de dispersión de energías EDS (JM LINK). El límite de detección del EDS es del rango de $0,1-1 \%$ peso en función del elemento que se desee analizar.

Después de recubiertas las probetas, se expusieron en un loop de vapor de agua a $750{ }^{\circ} \mathrm{C}$ en un ambiente donde el vapor de agua fue transportado hacia las muestras mediante un flujo continuo de agua a una velocidad 2,4 $\mathrm{mL} / \mathrm{min}$, más un flujo de gas de $\mathrm{N}_{2}$ de $40 \mathrm{~mL} / \mathrm{min}$. La exposición fue llevada hasta 1000 horas, sacando tres probetas a $200,400,600,800$ y 1000 horas. Una vez terminados los ensayos de oxidación a alta temperatura se procedió a caracterizar mecánicamente los recubrimientos oxidados y sin oxidar por medio de ensayos de micro dureza Vickers en un durómetro marca Wilson Wolper, utilizando una carga de $1000 \mathrm{gf}$ [20]. Se realizaron grupos de cinco indentaciones en cada punto, en diferentes áreas de la sección transversal de las muestras, tal como se ilustra en la Fig. 1, con el fin de conocer la microdureza en zonas importantes como el recubrimiento, la frontera entre el (recubrimiento-sustrato) y el sustrato, y así levantar un perfil de dureza.

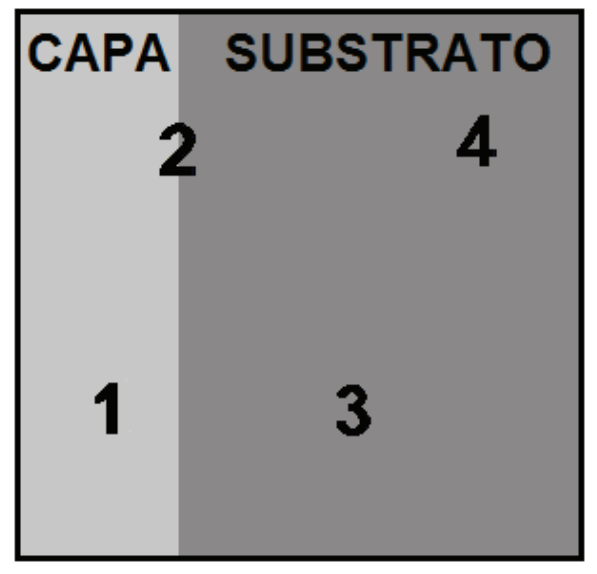

Fig. 1. Áreas seleccionadas para ensayos de dureza Vickers (Corte transversal).

La AFM se realizó en un equipo marca Nanosurf, utilizando el software easyScan 2 [21], y las imágenes fueron procesadas con el software SPIP 6.2.6. [22]. Para todas las pruebas de AFM, el sentido del barrido fue de izquierda a derecha, llevando el siguiente orden: Recubrimiento - Frontera (R-S) - sustrato. Tambien se realizaron ensayos de nanoindentación en un equipo Ibis Authority de la empresa Fischer Cripps Labs. El equipo se operó en modo de lazo cerrado; se programaron ciclos de carga-descarga, incluyendo periodos de 5 segundos y 15 segundos a carga máxima y a carga mínima, respectivamente, para tener en cuenta posibles efectos de Creep y variación térmica. La carga máxima fue de $1 \mathrm{mN}$, y se realizaron perfiles de dureza desde una distancia de aproximadamente 15 micras de la superficie del recubirmiento hasta el sustrato con pasos de 2 micras cerca a la intercara capa-sustrato y de 10 micras en el sustrato. El equipo 
fue previamente calibrado, y los resultados, analizados de acuerdo con el método de Oliver y Pharr [23].

\section{Resultados Y ANÁlisis}

En la Fig. 2 se observa el corte transversal para el acero AISI 317 recubierto con aluminio por CVDFBR, para una temperatura de deposición de $580{ }^{\circ} \mathrm{C}$. Según los análisis EDS $[5,11]$, los recubrimientos están compuestos, aproximadamente, por un $68 \%$ de aluminio, $20 \%$ de hierro, $9 \%$ de cromo y $3 \%$ de níquel. Se encontró que en la superficie hay mayor cantidad de aluminio y disminuye levemente hacia la interfase substrato-recubrimiento; por otra parte, el hierro y el cromo están en mayor porcentaje en la interfase substrato- recubrimiento que en la superficie del recubrimiento, mientras que el níquel está distribuido homogéneamente en el recubrimiento. Las capas tienen un espesor de aproximadamente 30 micras.

En la Tabla 1 se muestran las microdurezas Vickers de la sección transversal de los recubrimientos de aluminio depositados por CVD-FBR. Se observa que las microdurezas más altas se encuentran en la interfase recubrimiento-sustrato (zona 2), esto se debe a la presencia de intermetálicos de aluminio duros como $\mathrm{FeAl}, \mathrm{Fe}_{2} \mathrm{Al}_{5}$ y $\mathrm{Fe}_{2} \mathrm{AlCr}$ [2, 5, 7, 11]. Esto se debe a que durante el tratamiento térmico y el proceso de oxidación, el aluminio del recubrimiento difunde hacia el interior del sustrato, formando intermetálicos de aluminio que son más duros que el sustrato; esto fue observado en otros trabajos [7-9, $11]$.

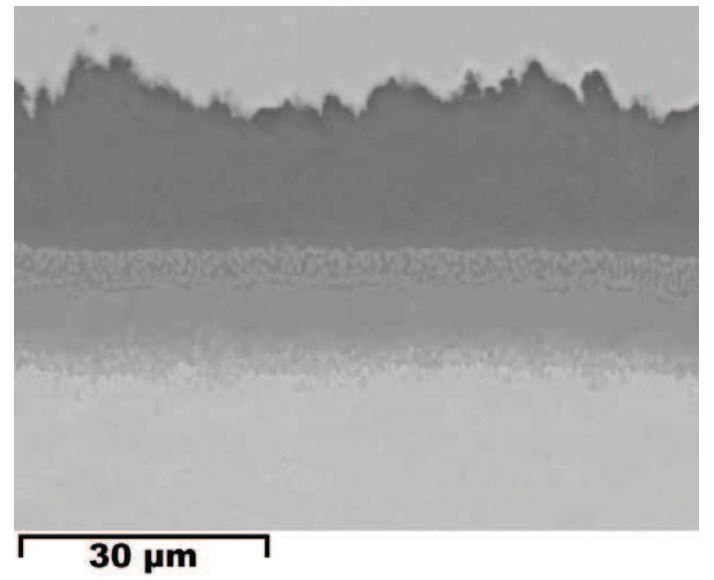

Fig. 2. Corte transversal del recubrimiento de aluminio depositado sobre acero inoxidable austenítico AISI 317.
Lo anterior podría ser un indicador de que el recubrimiento tiene buena adherencia al substrato, ya que en la interfase recubrimiento-sustrato no hay desprendimiento del recubrimiento, debido al ensayo de microdureza; además, se podría inferir que el recubrimiento tendría mejores propiedades mecánicas que el sustrato. Sin embargo, las durezas están subestimadas, ya que la diagonal de la huella es de aproximadamente 9 a 10 micras, que comparada con el espesor de la capa (30 micras, aproximadamente) no da un volumen de soporte adecuado a la indentación, lo que quiere decir que tanto en la capa como en la interfase hay una influencia del montaje metalográfico en la dureza medida; esto se corroborará más adelante con las mediciones de nanoindentación.

\section{TABLA 1}

DUREZA VICKERS DE LA SECCIÓN TRANSVERSAL DE LOS RECUBRIMIENTOS DE ALUMINIO

\begin{tabular}{|l|c|c|c|c|}
\hline & Sin TT & Con TT & $\begin{array}{c}\text { Oxidado } \\
\mathbf{6 0 0} \mathbf{~ h}\end{array}$ & $\begin{array}{c}\text { Oxidado } \\
\mathbf{1 0 0 0} \mathbf{~ h}\end{array}$ \\
\hline Zona 1 & 187,5 & 216,4 & 217,6 & 238,9 \\
\hline Zona 2 & 272,2 & 335,2 & 355,2 & 320,1 \\
\hline Zona 3 & 185,6 & 208,1 & 220.9 & 232,1 \\
\hline Zona 4 & 189,7 & 201,6 & 213.1 & 208,9 \\
\hline
\end{tabular}

Luego de realizar las microdurezas Vickers, se hicieron barridos con AFM, para obtener imágenes de la topografía de la sección transversal de la muestra, observando el estado del recubrimiento, de la frontera (recubrimiento-sustrato) y del sustrato. El AFM proporcionó información acerca del gradiente de voltaje que se genera al realizar los barridos, al ser utilizado en modo Contrast Phase [15, 24] (gráficos de Voltaje vs. Distancia recorrida por la punta del cantilever en los barridos), dejando ver que el gradiente de voltaje tiene su pico más alto en la zona del recubrimiento (Al) y disminuye gradualmente hasta estabilizarse a medida que se acerca al sustrato; en la Fig. 3 se muestra claramente lo antes mencionado. También se observó que las muestras que fueron sometidas a oxidación presentan una amplitud en la fase del barrido en un rango de 0,15-0,2 [V], mientras que las muestras que no fueron sometidas a oxidación manejan un rango menor $0,1-0,15[\mathrm{~V}]$. 

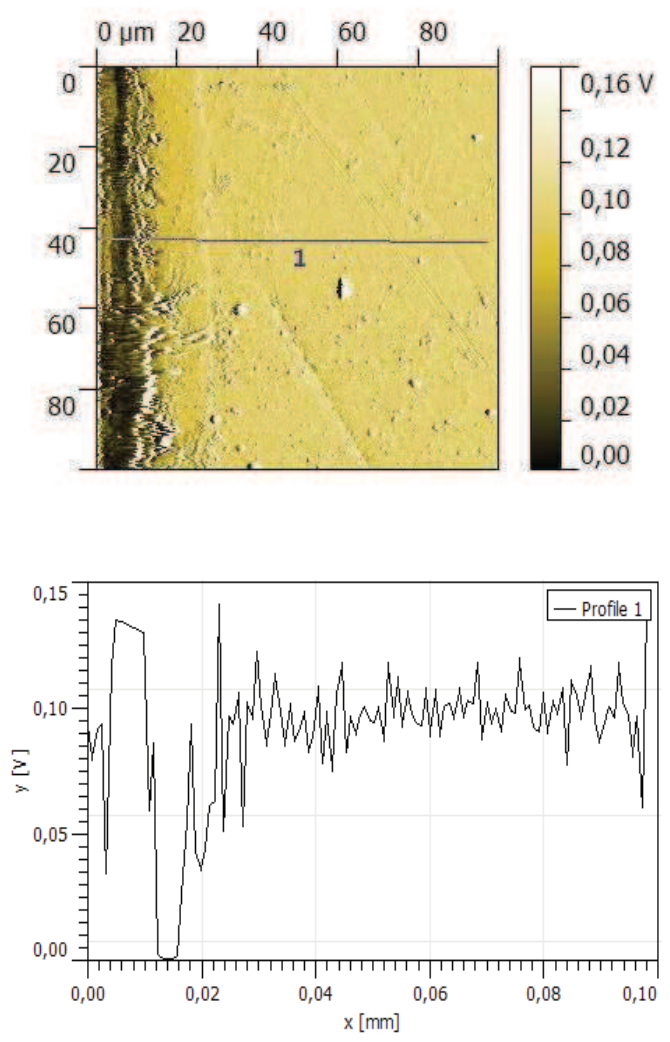

Fig. 3. Barrido AFM en modo Constrast Phase.

En la Fig. 4 se aprecian diferentes tonalidades de colores; las zonas superficiales de la muestra que presentan los picos más altos, de acuerdo con la amplitud de fase, están en color verde o rojo, y las zonas en donde la amplitud de frecuencia tiene sus puntos más bajos están en de color azul. Cuando la amplitud logra estabilizarse tiene un color marrón, que es precisamente cuando llega al sustrato. Este análisis de colores permite tener una idea visual del gradiente de amplitud de fase y facilita también una posible relación de dicha amplitud con otras propiedades como la dureza del recubrimiento y del sustrato. Se aprecian claramente picos más altos al principio del barrido (izquierda); esta área corresponde al recubrimiento de aluminio; luego, los picos bajan gradualmente hasta estabilizarse llegando al sustrato.

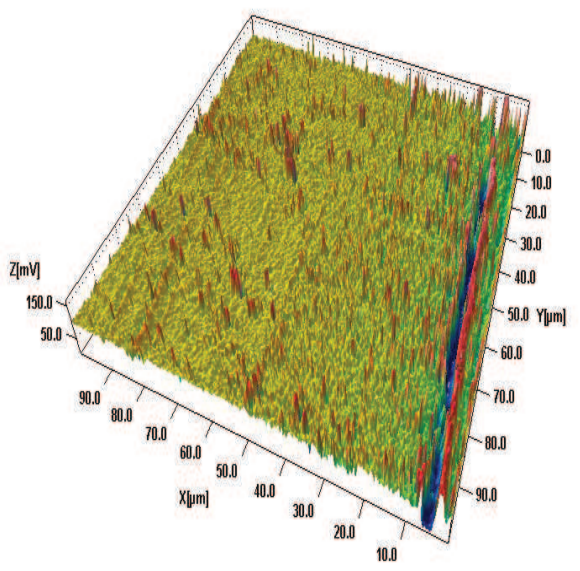

Fig. 4. Amplitud vs. Distancia para acero AISI 317, en tres dimensiones.

Finalmente, las muestras fueron sometidas a ensayos de nanoindentación, los cuales corroboraron los resultados obtenidos en las pruebas Vickers, y también están de acuerdo con los supuestos generados a partir de los gradientes de voltaje obtenidos en los barridos AFM. Los ensayos de nanoindentación mostraron un comportamiento descendente en la nanodureza de la muestra, teniendo su mayor valor en el área del recubrimiento, y también se aprecian valores estables cuando las mediciones se hicieron en el sustrato. Estos resultados son coherentes con los obtenidos en las pruebas de dureza Vickers y muestran, también, una relación con el gradiente de voltaje obtenido en los ensayos realizados con el Microscopio de Fuerza Atómica AFM.

En la Fig. 5 se puede apreciar el comportamiento de la nanodureza; esta figura muestra irregularidad en el área del recubrimiento, posiblemente, debido a la falta de uso de una placa para el soporte de la capa, por ello su pulido es un poco deficiente. Debido a estas irregularidades, la muestra presentó datos poco confiables en la zona exterior del recubrimiento, pero a medida que se avanza hacia el sustrato los datos son más coherentes y confiables; por tal motivo, los datos de nanodureza comienzan a $18 \mu \mathrm{m}$. 


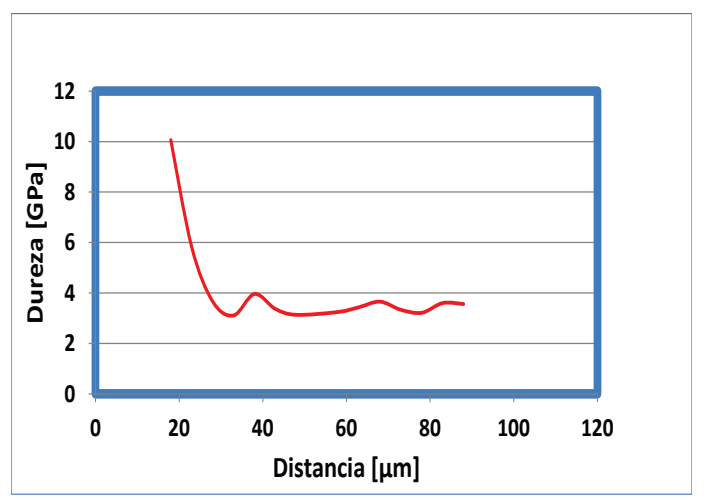

Fig. 5. Comportamiento de la nanodureza (Recubrimiento - Sustrato).

Las gráficas mostradas a continuación dejan ver el incremento de la dureza en el recubrimiento con respecto al sustrato. El aumento en la dureza es poco, pero es suficiente para observar un aporte notable del recubrimiento a la dureza del material.

El módulo de Young se comportó de manera muy similar a la dureza; tuvo su mayor valor en la zona del recubrimiento y presentó una disminución gradual a medida que se acercaba al sustrato, en donde finalmente se estabiliza. Aunque el cambio sea pequeño, es suficiente para concluir que el recubrimiento sí aporta a la capacidad elástica de la superficie del material, ayudando así a su rendimiento mecánico; la Fig. 6 enseña este comportamiento.

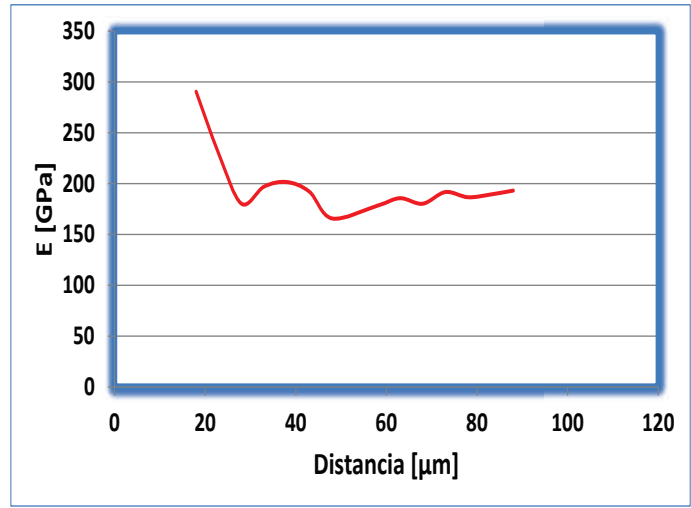

Fig. 6. Comportamiento del módulo de Young (Recubrimiento - Sustrato).

\section{Conclusiones}

Los datos arrojados por los ensayos de dureza Vickers muestran claramente un aumento en la dureza del recubrimiento con respecto al sustrato.

Las gráficas e imágenes obtenidas con los barridos realizados con el AFM dejan ver un gradiente en la variación de la amplitud de fase para la mayoría de las muestras. Dichos gradientes, al ser correlacionados con los datos obtenidos en los ensayos Vickers y, posteriormente, en los ensayos de Nanoindentación, expresan una estrecha relación entre dicha amplitud y la dureza en la superficie de la muestra (RecubrimientoSustrato).

En estudios anteriores se demostró que los recubrimientos evaluados aumentan la resistencia a la oxidación del sustrato; por tal condición se espera un buen desempeño del acero recubierto bajo las condiciones de temperatura y oxidación a las cuales fueron sometidas.

La continuidad entre el recubrimiento y el sustrato, evidenciada mediante el SEM y el AFM, permite inferir que hay buena adhesión entre las dos fases; además, los datos de dureza en la interfase recubrimiento-sustrato son buenos.

Se logró comprobar una notable mejoría en las propiedades mecánicas del sustrato bajo las condiciones de evaluación.

\section{REFERENCIAS}

[1] R. Trevisan, C. Lima, Aspersao Termica Fundamentos E Aplicacoes. Brasil; Artliber. 2002.

[2] J. L. Marulanda, S.I. Castañeda-Quintana, \& A. Remolina-Millan, "Recubrimientos depositados por CVD-FBR para protección a alta temperatura". Dyna; vol. 80, n. ${ }^{\circ} 181$, pp. 181-91. 2013.

[3] J. Pérez-Mariano, J. Caro, C. Colominas. "Multilayer Coatings by Chemical Vapor Deposition in a Fluidized Bed Reactor at Atmospheric Pressure (Ap/Fbr-Cvd): Tin/Tan And Tin/W". Surface \& Coatings Technology. Elsevier, vol. 201, no. 6, pp. 2174-2180. 2006. 
Caracterización mecánica de recubrimientos de aluminio por CVD-FBR sobre aceros inoxidables y resistencia a la oxidación en vapor de agua

Disponible en: http://www.sciencedirect.com/ science/article/pii/S025789720600260X

[4] S. Hogmark, S. Jacobson y M. Larsson. Wear, vol. 246, pp. 20-33. 2000.

[5] J.L. Marulanda-Arévalo, F. J.Pérez-Trujillo, \& S. I. Castañeda-Quintana, "Behavior of aluminum coating by CVD-FBR in steam oxidation at 700 ${ }^{\circ} \mathrm{C}$ '. CT\&F - Ciencia, Tecnología y Futuro, vol. 5, n. ${ }^{\circ}$ 4, pp. 77-86. 2014.

[6] J. Pérez-Mariano, K.H. Lau, A. Sanjurjo, J. Caro, D. Casellas, C. Colominas. "TiSiN nanocomposite coatings by chemical vapor deposition in a fluidized bed reactor at atmospheric pressure (AP/FBR-CVD)", Surface and Coatings Technology, vol. 201, n. ${ }^{\circ}$ 6, pp. 2217-2225, 2006.

[7] J. L. Marulanda-Arévalo, F. J. Pérez-Trujillo y A. Remolina-Millán, "Recubrimientos de aluminio-silicio realizados por deposición química de vapor en lecho fluidizado sobre el acero inoxidable AISI 316". Revista Facultad de Ingeniería, Uptc, Vol. 22, N. ${ }^{\circ}$ 34. Enero-Junio de 2013.

[8] P. Rodríguez, B. Caussat, C. Ablitzer, X. Iltis, $\&$ M. Brothier. "Fluidization and coating of very dense powders by Fluidized Bed Chemical Vapour Deposition". Chemical Engineering Research and Design, vol. 91, pp. 2477-2483. 2013.

[9] F.J. Pérez, F. Pedraza, M. P. Hierro and, P.Y. Hou, "Adhesion properties of aluminide coatings deposited via CVD in fluidised bed reactors (CVD-FBR) on AISI 304 stainless steel", Surface \& Coatings Technology, vol. 133-134, pp. 338-343. 2000. Disponible en: http://dx.doi. org/10.1016/S0257-8972(00)00952-X.

[10] G. Angelopoulos, S. Kinkel, N. Voudouris, Surface and Coatings Technology, vol. 78, pp. 72-77. 1996.

[11] J.L. Marulanda-Arévalo, S.I. CastañedaQuintana y A. Remolina-Millán, "Oxidación en vapor de agua a $700{ }^{\circ} \mathrm{C}$ y $750{ }^{\circ} \mathrm{C}$ del acero inoxidable AISI 317 recubierto con aluminio por CVD-FBR”. Rev. Fac. Ing. Univ. Antioquia N. ${ }^{\circ}$ 71, pp. 191-201. 2014.

[12] D.Pérez-Muñoz, Nanocaracterización mecánica de recubrimientos de aluminio depositados sobre aceros inoxidables austeníticos sometidos a oxidación en vapor de agua. Tesis pregrado en Ingeniería Mecánica. Colombia. Universidad tecnológica de Pereira. Facultad de Ingeniería Mecánica. 2014.

[13] M. Salerno, N. Patra \& A. Diaspro. "Atomic force microscopy nanoindentation of a dental restorative midifill composite". Dental Materials, vol. 28, pp. 197-203. 2012.

[14] Electrical Mapping of AISI 304 Stainless Steel Subjected to Intergranular Corrison Performed by Means of AFM - L.

[15] Y. Xia, M. Bigerelle, S. Bouvier, A. Lost, P.-E. Mazeran. Quantitative Approach to Determine the Mechanical Properties by Nanoindentation Test: Application on Sandblasted Materials. February 2015, pages 297-304.

[16] Reza Pejman, S. Hassan Salehi, Manouchehr Salehi. "Numerical study of interfacial crack growth effects on Nanoindentation mechanical properties in presence of pre-existing defect". October 2013, pp. 408-413.

[17] Fisher-Cripps, Anthony C. Nanoindentation, Mechanical Engineering Series, $2^{\text {nd }}$ edition, New York: Springer. 2012.

[18] A. Stylianou \& D. Yova. "Surface nanoscale imaging of collagen thin films by Atomic Force Microscopy". Materials Science and Engineering, C, Materials for biological applications vol. 33, no. 5, pp. 2947-2957. 2013.

[19] B. Destouches, Estudio del efecto del tamaño en nanoindentación. Tesis Maestría en Biomateriales. España. Universidad Politécnica de Cataluña. Departamento de Ciencia de los Materiales. 2008.

[20] ASTM Standards, Estándar para ensayo de dureza Rockwell y dureza superficial Rockwell de materiales metálicos. ASTM E18-03.

[21] Nanosurf. Easyscan 2 AFM Operating Instructions, SPM Control Software, versión 3.0. July 2011.

[22] Image Metrology. SPIP Handbook, Control Software, version 6.2.6, 2014.

[23] Qianhua Kan, Wenyi Yan, Guozheng Kang, Qingping Su. "Oliver-Pharr indentation method in determining elastic moduli of shape memory alloys - A phase transformable material". Journal PF the Mechanics and Physics of Solids, Vol. 61, no. 10, pp. 2015-2033, October 2013.

[24] L. E. Bertassoni, G. W. Marshall, M. V. Swain. "Mechanical heterogeneity of dentin at different length scales as determined by AFM phase contrast". Micron, Vol. 43, no. 12, pp. 13641371, December 2012. 